\title{
miR-145-5p inhibits epithelial-mesenchymal transition via the JNK signaling pathway by targeting MAP3K1 in non-small cell lung cancer cells
}

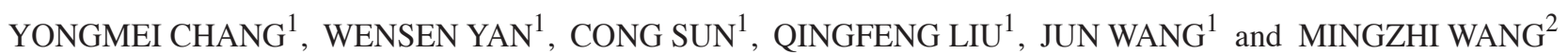 \\ Departments of ${ }^{1}$ Respiratory Medicine and ${ }^{2}$ Cardiothoracic Surgery, \\ Guangdong No. 2 Provincial People Hospital, Guangzhou, Guangdong 510317, P.R. China
}

Received January 8, 2016; Accepted July 3, 2017

DOI: $10.3892 / \mathrm{ol} .2017 .7092$

\begin{abstract}
Lung cancer is one of the most common types of tumors and the leading cause of cancer-associated mortality in the world. Additionally, non-small cell lung cancer (NSCLC) accounts for $\sim 80 \%$ of all lung cancer cases. Epithelialmesenchymal transition (EMT) is an important cell biological process, which is associated with cancer migration, metastasis, asthma and fibrosis in the lung. In the present study, it was revealed that miR-145-5p was able to suppress EMT by inactivating the c-Jun N-terminal kinase (JNK) signaling pathway in NSCLC cells. Mitogen-activated protein kinase kinase kinase 1 (MAP3K1) was predicted and confirmed to be a novel target of miR-145-5p. Overexpression of MAP3K1 was able to reverse the inhibition of EMT induced by miR-145-5p via the JNK signaling pathway. Overall, the results revealed that miR-145-5p inhibits EMT via the JNK signaling pathway by targeting MAP3K1 in NSCLC cells.
\end{abstract}

\section{Introduction}

Lung cancer is one of the most common types of tumors and the leading cause of cancer-associated mortality in the world (1). Non-small cell lung cancer (NSCLC) accounts for $\sim 80 \%$ of all lung cancer cases (2). Although many advances have been achieved in diagnosis and treatment and progress

Correspondence to: Dr Mingzhi Wang, Department of Cardiothoracic Surgery, Guangdong No. 2 Provincial People Hospital, 1 Shiliugang Road, Guangzhou, Guangdong 510317, P.R. China

E-mail:wmz891@163.com

Dr Jun Wang, Department of Respiratory Medicine, Guangdong No. 2 Provincial People Hospital, 1 Shiliugang Road, Guangzhou, Guangdong 510317, P.R. China

E-mail: shandongrenwang1@163.com

Keywords: miR-145-5p, epithelial-mesenchymaltransition, mitogenactivated protein kinase kinase kinase 1, Jun N-terminal kinase 2, non-small cell lung cancer has been made in lung cancer therapy, there is $~ 5 \%$ improvement in 5-year survival rates for the previous 20 years (3).

Epithelial-mesenchymal transition (EMT) is an important cell biological process, which is associated with cancer migration, metastasis, asthma and fibrosis in the lung (4). When EMT occurs, epithelial cells gradually transform into mesenchymal-like cells by losing their epithelial-associated functions and characteristics (5). Epigenetic downregulation of E-cadherin expression was observed in advanced NSCLC and restoration of E-cadherin expression strongly suppressed the invasion/migration of tumor cells (6,7). Several signaling pathways are involved in EMT in NSCLC, including TGF- $\beta$ (8), Slug (9), Wnt/ $\beta$-catenin/zinc finger E-box binding homeobox 1 signaling (10) and c-Jun N-terminal kinase (JNK) signaling (11).

MicroRNAs (miRNAs) are small non-coding RNAs, which regulate gene expression post-transcriptionally. miRNAs are involved in the pathogenesis of lung diseases, including lung cancer, cystic fibrosis, chronic obstructive pulmonary disease, asthma and idiopathic pulmonary fibrosis (12). miRNAs contribute to the development of novel diagnostic biomarkers and personalized therapeutic tools in NSCLC (13).

miR-145 is silenced by DNA methylation and acts as a prognostic biomarker in NSCLC (14). miR-145 regulates chemoresistance in hepatocellular carcinoma (15) and bladder cancer (16) via EMT. miR-145 inhibits migration and invasion by down-regulating fascin actin-bundling protein 1 (FSCN1) (17) and mucin 1 (18).

The aim of the present study was to identify the function of miR-145-5p in EMT in NSCLC cells. In addition, it was investigated whether the target of miR-145-5p mediated this process. The findings of the present study will provide new insights into the functions of miR-145-5p as well as its molecular mechanisms in NSCLC.

\section{Materials and methods}

Cell culture and treatment. The NSCLC cell lines A549 and H520, and the normal lung bronchus epithelial cell line 16HBE were obtained from American Type Culture Collection (Manassas, VA, USA). The cells were grown in Dulbecco's modified Eagle's medium supplemented with $10 \%$ fetal bovine 
serum, $2 \mu \mathrm{M}$ glutamine, $100 \mathrm{IU} / \mathrm{ml}$ penicillin, and $100 \mu \mathrm{g} / \mathrm{ml}$ streptomycin sulfate and were cultured in a humidified chamber with $5 \% \mathrm{CO}_{2}$ at $37^{\circ} \mathrm{C}$. All transfections were performed using Lipofectamine 2000 (Invitrogen; Thermo Fisher Scientific, Inc., Waltham, MA, USA). In order to interrupt the MAP3K1 mediated signaling pathway, cells were treated with $30 \mu \mathrm{m}$ SP100625 (Enzo Life Sciences, Inc., Farmingdale, NY, USA) for $24 \mathrm{~h}$.

Target prediction of miR-145-5p by Targetscan. Targetscan (http://www.targetscan.org/vert_71/) is an online software for the prediction of the binding of miRNAs to target genes. miR-145-5p investigated using the software to search for its target genes.

Transfection and apoptosis assay. The cells were placed in six-well plates at $70-90 \%$ confluence $24 \mathrm{~h}$ prior to transfection in serum-free media. The cells were transfected with plasmids, pcDNA3.1 or pcDNA3.1-MAP3K $1(2 \mu \mathrm{g} / \mathrm{well}$; Fulengen, Guangzhou, China; http://www.fulengen.com/) or miRNA/small-interfering RNA (100 pmol/well) using Lipofectamine 2000, according to the manufacture's protocol. After $48 \mathrm{~h}$, the cells were collected by trypsinization, washed with phosphate-buffered saline and used for subsequent experiments.

Luciferase reporter assay. The entire 3'-untranslated region (UTR) of human mitogen-activated protein kinase kinase kinase 1 (MAP3K1) was cloned by Genscript (Nanjing, China; http://www.genscript.com.cn/). The entire 3'-UTR of MAP3K1 were inserted into the pMIR-reporter plasmid (Ambion; Thermo Fisher Scientific, Inc.). The insertion was confirmed to be correct by DNA sequencing. The sequences that interact with the seed sequences of miR-145-5p were mutated. Luciferase assays were performed using A549 cells. The cells were transfected with: miR-145-5p mimics, 5'-GUC CAGUUUUCCCAGGAAUCCCU-3' or scrambled sequences, 5'-UUCUCCGAACGUGUCACGUTT-3'; GenePharm, Inc. (Sunnyvale, CA, USA) and Renilla luciferase plasmids (Ambion; Thermo Fisher Scientific, Inc.) in 24-well plates. The cells were lysed for luciferase assay $48 \mathrm{~h}$ following transfection. Luciferase assays were performed using dual luciferase assay kit (Promega Corporation, Madison, WI, USA) according to the manufacturer's protocol.

Reverse transcription quantitative polymerase chain reaction $(R T-q P C R)$. Total RNA was extracted from NSCLC cell lines and 16HBE cells using TRIzol (Thermo Fisher Scientific, Inc.). Complementary DNAs were synthesized by using specific-miRNA primers (GeneCopoeia Inc., Guangzhou, China). The primers of miR-145-5p (HmiRQP0192) were obtained from Fulengen. U6 snRNA was used as an internal control. Gene expression levels were measured by RT-qPCR using BioRad CFX (Bio-Rad Laboratories, Inc., Hercules, CA, USA) and SYBR-Green kit (Takara Biotechnology Co., Ltd., Dalian, China). Reactions were incubated for $10 \mathrm{~min}$ at $95^{\circ} \mathrm{C}$, followed by 40 cycles of $95^{\circ} \mathrm{C}$ for $10 \mathrm{sec} ; 60^{\circ} \mathrm{C}$ for $20 \mathrm{sec}$ and $72^{\circ} \mathrm{C}$ for $10 \mathrm{sec}$. The primers of were as follows: MAP3K1 forward, 5'-TAGGGCCTAACTCTTTCCTGAT-3' and reverse, 5'-GTTCTAGTTGAAACACCCGGA-3'; GAPDH forward,
5'-CAATGACCCCTTCATTGACC-3' and reverse, 5'-GAC AAGCTTCCCGTTCTCAG-3'. GAPDH was used as endogenous control to normalize the expression data. Reactions were incubated for $10 \mathrm{~min}$ at $95^{\circ} \mathrm{C}$, followed by 40 cycles of $95^{\circ} \mathrm{C}$ for $10 \mathrm{sec} ; 60^{\circ} \mathrm{C}$ for $20 \mathrm{sec}$ and $72^{\circ} \mathrm{C}$ for $15 \mathrm{sec}$. Each sample was analyzed in quadruplicate. The relative amount of miRNA was normalized to U6: Forward, 5'-CTCGCTTCGGCAG CACA-3' and reverse, 5'-AACGCTTCACGAATTTGCGT-3'. The data was calculated with the equation $2^{-\Delta \Delta C q}$, where $\Delta \Delta \mathrm{Cq}=\left(\mathrm{Cq}_{\mathrm{miRNA}}-\mathrm{Cq}_{\mathrm{U} 6}\right)$ target $-\left(\mathrm{Cq}_{\mathrm{miRNA}}-\mathrm{Cq}_{\mathrm{U} 6}\right)$ control. Gene expression was calculated with the equation $2^{-\Delta \Delta \mathrm{Cq}}$, where ${ }^{\Delta \Delta} \mathrm{Cq}=\left(\mathrm{Cq}_{\text {target }}-\mathrm{Cq}_{\mathrm{GAPDH}}\right)-\left(\mathrm{Cq}_{\text {control }}-\mathrm{Cq}_{\mathrm{GAPDH}}\right)(19)$.

Western blotting. The cells were suspended in radioimmunoprecipitation assay protein lysis buffer ( $\mathrm{pH}$ 7.4), containing $20 \mathrm{mM}$ sodium phosphate, $150 \mathrm{mM}$ sodium chloride, $5 \mathrm{mM}$ EDTA, $5 \mathrm{mM}$ phenylmethylsulfonyl fluoride, $1 \%$ aprotinin, $1 \mu \mathrm{g} / \mathrm{ml}$ leupeptin, and $500 \mu \mathrm{M} \mathrm{Na}_{3} \mathrm{VO}_{4}$. Protein concentration was quantified using Pierce BCA protein assay kit (Thermo Fisher Scientific, Inc.). Total protein $(50 \mu \mathrm{g})$ was resolved with $6 \%$ SDS-PAGE, and transferred to a polyvinylidene difluoride membrane. Membranes were blocked in 5\% non-fat milk diluted in TBST for $1 \mathrm{~h}$ at room temperature. The blots were probed with primary antibodies against MAP3K1 (cat. no. PA5-15085; Invitrogen; Thermo Fisher Scientific, Inc.), E-cadherin (cat. no. 14472), Vimentin (cat. no. 5741), JNK (cat. no. 9252), p-JNK (cat. no. 9255), GAPDH (cat. no. 5174) all purchased from Cell Signaling Technology, Inc., Danvers, MA, USA. All primary antibodies were diluted at 1:1,000 and incubated at $4^{\circ} \mathrm{C}$ overnight. After washing, membranes were probed with appropriate secondary antibodies, horseradish peroxidase conjugated-goat anti mouse IgG (cat. no. BA1051; Boster Biological Technology, Pleasanton, CA, USA) or horseradish peroxidase conjugated-goat anti rabbit IgG (cat. no. BA1054; Boster Biological Technology). Secondary antibodies were diluted at 1:5,000 and incubated at room temperature for $1 \mathrm{~h}$. All membranes were stripped by incubating in Restore PLUS Western blot stripping buffer (Thermo Fisher Scientific, Inc.) for $15 \mathrm{~min}$ at room temperature and reprobed with anti-GAPDH antibody for loading control. The blots were visualized using enhanced chemiluminescence (GE Healthcare, Chicago, IL, USA).

Statistical analysis. Statistical analysis was performed with SPSS17.0 (SPSS, Inc., Chicago, IL, USA). The Student's t-test was used to compare the different groups. $\mathrm{P}<0.05$ was considered to indicate a statistically significant difference, and P-values are represented by an asterisk on the bars in the figures.

\section{Results}

Ectopic expression of miR-145-5p inhibits EMT in NSCLC cells. The effect of miR-145-5p mimics and inhibitor transfection was detected (Fig. 1A). It was demonstrated that ectopic expression of miR-145-5p markedly increased the expression of E-cadherin and suppressed the expression of vimentin in A549 and H520 cells, compared with mi-NC. By contrast, depletion of miR-145-5p induced E-cadherin downregulation and vimentin upregulation in A549 and H520 cells (Fig. 1B). 
A
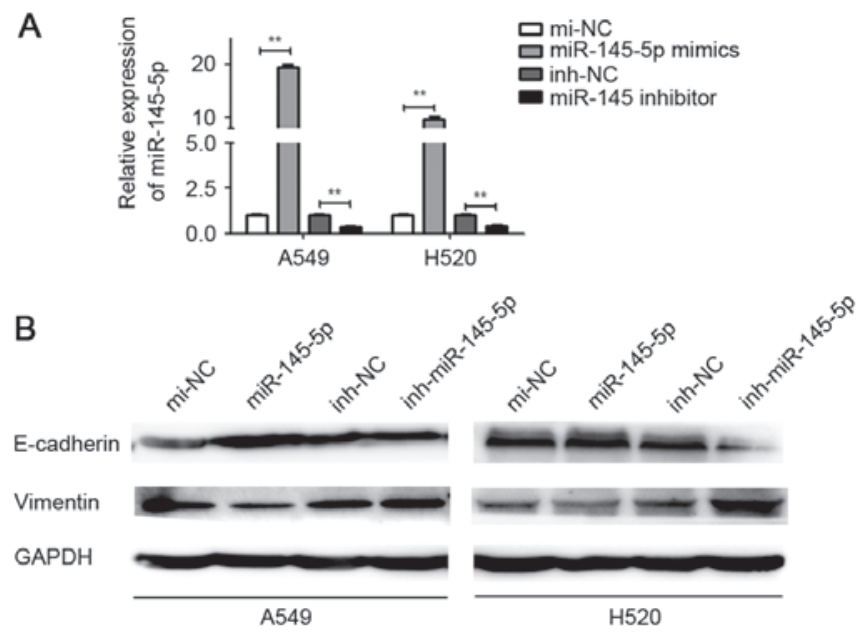

Figure 1. miR-145-5p regulates the expression of EMT-associated genes in non-small cell lung cancer cells. (A) Graph displaying results from quantitative reverse transcription polymerase chain reaction analysis following transfection of A549 and H520 cells with miR-145-5p mimics or inhibitor. The values (means \pm standard deviation) indicate the relative expression of miR-145-5p in each group. Results are expressed as the mean \pm standard deviation of three independent experiments. ${ }^{* *} \mathrm{P}<0.01$. (B) Effect of miR-145-5p on E-cadherin and vimentin expression in A549 and H520 cells as determined by western blotting. GAPDH was used as the loading control. NC, negative control; miRNA, microRNA; inh, inhibitor.

Involvement of JNK signaling in miR-145-5p-induced EMT suppression in NSCLC. JNK is not required for initiation of NSCLC, but associated with EMT. JNK drives transcription of numerous key EMT genes (20). It has been reported that TGF- $\beta 1$ induced JNK activation during EMT in idiopathic pulmonary fibrosis (21). In the present study, it was examined whether JNK signaling pathway has a critical role in the regulation of miR-145-5p-mediated EMT in NSCLC cells. The results revealed that overexpression of miR-145-5p was able to inhibit the expression of phosphorylated (p)-JNK, but has no effect on the level of total JNK protein (Fig. 2A). The cells were also treated with a combination of a specific JNK inhibitor (SP600125) and miR-145-5p. Western blotting analysis showed SP600125 was able to reverse the effect of miR-145-5p on EMT in A549 and H520 cells (Fig. 2B).

MAP3K1 is a target of miR-145-5p. TargetScan online software was used to search for putative protein-coding gene targets of miR-145-5p. The results indicated that MAP3K1 is a putative target of miR-145-5p (Fig. 3A). The MAP3K1 3'UTR was cloned into a luciferase reporter vector and binding sites of the mutant vector. The A549 cells were co-transfected with either mutated pmiR-MAP3K1-3'UTR and miR-145-5p mimics or wild-type pmiR-MAP3K1-3'UTR and miR-145-5p mimics. The cells were also co-transfected with mutated pmiR-MAP3K1 and negative control or wild-type pmiR-MAP3K1 and negative control. Luciferase activity was measured $48 \mathrm{~h}$ following transfection.

The results showed that the cells co-transfected with wild-type pmiR-MAP3K1-3'UTR and miR-145-5p mimic exhibited significant reduction in luciferase activity compared with cells cotransfected with the control. By contrast, the luciferase activity in cells transfected with mutated pmiR-MAP3K1-3'UTR and miR-145-5p mimic was not significantly altered (Fig. 3B).

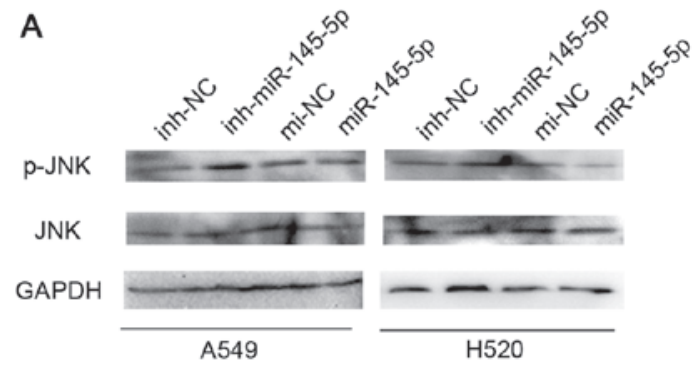

B

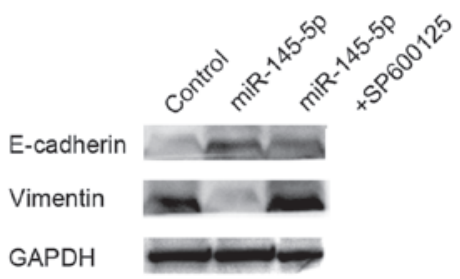

Figure 2. miR-145-5p regulates epithelial-mesenchymal transition via JNK signaling in NSCLC cells. (A) The effect of miR-145-5p on the JNK signaling pathway in A549 and $\mathrm{H} 520$ cells was determined by western blotting. GAPDH was used as the loading control. (B) The effect of miR-145-5p on E-cadherin and vimentin, which was mediated by JNK specific inhibitor SP600125 in A549 and H520 cells as determined by western blotting. GAPDH was used as the loading control. JNK, c-Jun N-terminal kinase; miRNA, microRNA; NC, negative control; inh, inhibitor.

Furthermore, RT-qPCR and western blotting analysis was used to investigate the expression of MAP3K1 regulated by miR-145-5p in A549 and H520 cells. The results showed that a marked reduction in mRNA and protein level of MAP3K1 in miR-145-5p-overexpressed A549 and H520 cells compared with the control (Fig. 3C and D).

MAP3K1 reverses miR-145-5p- induced inhibition of EMT in NSCLC. Since MAP3K1 was a novel target of miR-145-5p in NSCLC cells, it was investigated whether the effect of miR-145 on EMT was mediated by MAP3K1. Fig. 4A showed that the knockdown of MAP3K1 markedly induced the expression of E-cadherin and inhibited the expression of vimentin in A549 and $\mathrm{H} 520$ cells, while overexpression of MAP3K1 treatment reversed this effect. The ectopic expression of MAP3K1 was able to partly reverse the downregulation of p-JNK induced by the miR-145-5p inhibitor compared to the vector group. While, the suppression of MAP3K1 was able to interrupt the upregulation of p-JNK induced by miR-145-5p mimics compared to the siRNA negative control group (Fig. 4B).

\section{Discussion}

EMT is a characteristic feature of the majority of metastatic cells (22). Epithelial cells are highly differentiated, polarized and organized. When EMT occurs, these cells are transformed into undifferentiated, isolated and mesenchymal-like cells (23). E-cadherin and vimentin are known to be closely associated with EMT (24).E-cadherin has important functions in regulating cell-cell interactions (25). Epigenetic silencing of E-cadherin expression was observed in advanced NSCLC, and restoration of E-cadherin expression strongly suppressed the metastasis of cancer cells (7). Vimentin is specifically expressed in normal mesenchymal cells and overexpressed in lung cancer cells (26). In previous studies, vimentin has been 
A

Position 1791-1797 of MAP3K1 3' UTR

hsa-miR-145-5p
5'...CUUGCCAUUUUAUGUACUgGaAg...

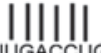

B

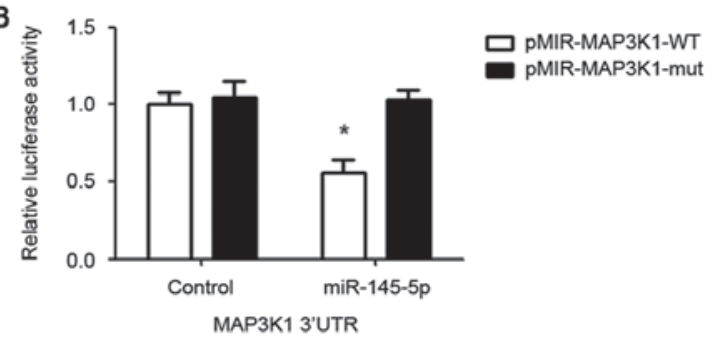

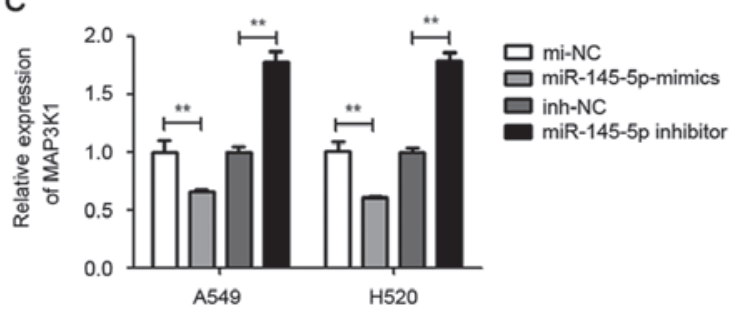

D

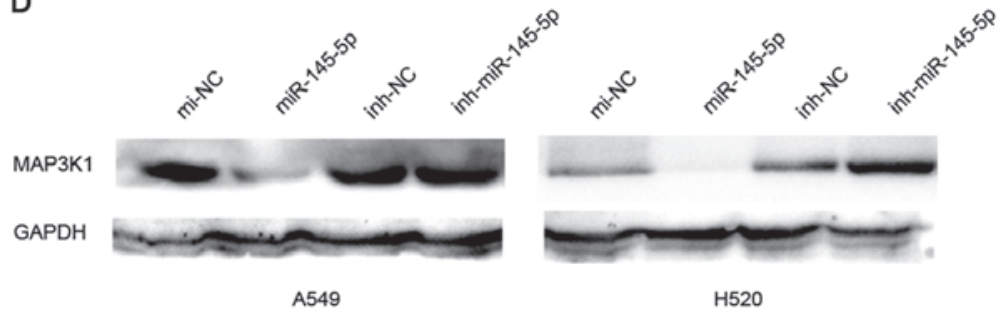

Figure 3. MAP3K1 is a novel marker of miR-145-5p. (A) Schematic representation of wild-type and mutated putative miR-145-5p-binding sites in the 3'UTR of MAP3K1. (B) A549 cells were co-transfected with wild-type or mutated MAP3K1 3'-UTR, pGL-3 control and either miR-145-5p or negative control mimics. Relative firefly luciferase activity was normalized to Renilla luciferase activity. Results are presented as the mean \pm standard deviation of three independent experiments. "P<0.01. (C) Expression of MAP3K1 mRNA was detected by quantitative reverse transcription polymerase chain reaction analysis in A549 and H520 cells transfected with miR-145-5p mimics, miR-145-5p inhibitor or control sequences. Results are presented as the mean \pm standard deviation of three independent experiments. ${ }^{* *} \mathrm{P}<0.01$. (D) Protein levels were detected by western blot analysis in A549 and H520 cells transfected with miR-145-5p mimics, miR-145-5p inhibitor or control sequences. GAPDH was used as an internal control. WT, wild-type; miRNA, microRNA; MAP3K1, mitogen-activated protein kinase 1; Mut, mutated; NC, negative control; UTR, untranslated region.

A

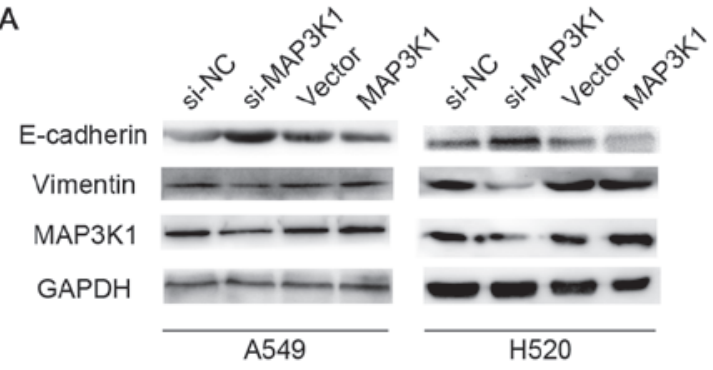

B

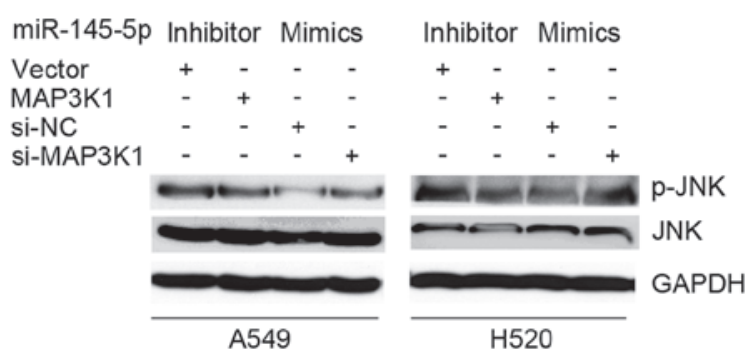

Figure 4. MAP3K1 mediates the regulation of the JNK signaling pathway via miR-145-5p in NSCLC cells. (A) Effect of MAP3K1 on the JNK signaling pathway in A549 and $\mathrm{H} 520$ cells was determined by western blotting. GAPDH was used as the loading control. (B) Effect of miR-145-5p on E-cadherin and vimentin, which mediated MAP3K1 expression in A549 and H520 cells was determined by western blotting. GAPDH was used as the loading control. JNK, c-Jun N-terminal kinase; MAP3K1, mitogen-activated protein kinase 1; miRNA, microRNA; p-, phosphorylated; si, small-interfering.

considered as a marker for EMT (26), and EMT is considered to be involved in the pathogenesis of lung cancer (5). Insulin like growth factor 1 receptor-induced resistance to epidermal growth factor receptor-tyrosine kinase inhibitors in advanced NSCLC is mediated by EMT (27). MARVELD1 suppresses EMT in NSCLC cells (28). Inactivation of M2 acetylcholine receptor/nuclear factor- $\kappa \mathrm{B}$ signaling axis reverses EMT and suppresses migration and invasion in NSCLC cells (29).

miR-145 was reported to inhibit metastasis in multiple tumors, including hepatocellular carcinoma (30), osteosarcoma (31) and nasopharyngeal carcinoma (32). miR-145 inhibits migration and invasion by downregulating FSCN1 (17) and mucin1 (18). A previous study by the present authors revealed that miR-145 acts as a metastasis suppressor by targeting metadherin in lung cancer (33). miR-145 regulates chemoresistance in hepatocellular carcinoma via EMT (15). However, the underlying mechanisms of miR-145-5p in regulating EMT in NSCLC are unclear. In the present investigation, it was demonstrated that miR-145-5p increased the expression of E-cadherin and suppressed the expression of vimentin. Therefore, it was hypothesized that miR-145-5p may inhibit metastasis via suppressing EMT in NSCLC cells.

The JNK signaling pathway exert an important role in the function of NSCLC cells. It was previously demonstrated that protein $4.1 \mathrm{~N}$, a member of the protein 4.1 family, was able to suppress metastasis by inactivating JNK-c-Jun signaling pathway in NSCLC (34). Celastrol induces cisplatin re-sensitization through inhibition of the JNK/activating transcription factor 2 signaling pathway in NSCLC (35). JNK signaling mediates EPH receptor A2-dependent tumor cell proliferation, motility and cancer stem cell-like properties in NSCLC cells (36). miR-145 induces apoptosis in ethanol-induced acute gastric mucosal injury via the JNK signaling pathway (37). However, no studies have yet shown the association between miR-145-5p and the JNK signaling pathway in NSCLC.

In the present study, the effect of miR-145-5p on JNK signaling pathway in NSCLC cells was detected. The results showed that miR-145-5p was able to inactivate the JNK signaling pathway in NSCLC cells. The results of the present study are in accordance with previous research from another group (38). 
MAP3K1 is a component of an evolutionarily conserved signal transduction cascade, which can activate mitogen-activated protein kinases (MAPKs) (39). It has been previously revealed that the single nucleotide polymorphism MAP3K1 rs889312 is closely associated with the risk of breast cancer and outcome among patients with diffuse-type gastric cancer (40).

In the present study, it was predicted and confirmed that MAP3K1 is a potential target of miR-145-5p. In order to clarify the function of MAP3K1 on EMT in NSCLC cells, the expression of E-cadherin and vimentin regulated by MAP3K1 was detected. The data illustrated that ectopic expression of MAP3K1 was able to inhibit E-cadherin protein levels and increase vimentin protein levels in NSCLC cells. Subsequently, whether MAP3K1 regulates ETM via a miR-145-5p mediated-JNK signaling pathway was investigated. The results revealed that overexpression of miR-145-5p and inhibition of MAP3K1 was able to reverse the expression of p-JNK in NSCLC cells. To conclude, miR-145-5p was able to inhibit EMT via JNK signaling pathway by targeting MAP3K1 in NSCLC cells.

\section{Acknowledgements}

The present study was supported by the Natural Science Foundation of Guangdong Province (grant no. 2016zc0160) and the Science and Technology Planning Project of Guangdong Province, China (grant no. 2014A020212447).

\section{References}

1. Hu J, Qiu M, Jiang F, Zhang S, Yang X, Wang J, Xu L and Yin R: MiR-145 regulates cancer stem-like properties and epithelial-to-mesenchymal transition in lung adenocarcinoma-initiating cells. Tumour Biol 35: 8953-8961, 2014.

2. Mariotto AB, Noone AM, Howlader N, Cho H, Keel GE, Garshell J, Woloshin S and Schwartz LM: Cancer survival: An overview of measures, uses, and interpretation. J Natl Cancer Inst Monogr 2014: 145-186, 2014.

3. Johnson DH, Schiller JH and Bunn PA Jr: Recent clinical advances in lung cancer management. J Clin Oncol 32: 973-982, 2014.

4. Tian L, Shen D, Li X, Shan X, Wang X, Yan Q and Liu J: Ginsenoside Rg3 inhibits epithelial-mesenchymal transition (EMT) and invasion of lung cancer by down-regulating FUT4. Oncotarget 7: 1619-1632, 2016.

5. Bartis D, Mise N, Mahida RY, Eickelberg O and Thickett DR: Epithelial-mesenchymal transition in lung development and disease: Does it exist and is it important? Thorax 69: 760-765, 2014.

6. Yuan X, Wu H, Han N, Xu H, Chu Q, Yu S, Chen Y and Wu K: Notch signaling and EMT in non-small cell lung cancer: Biological significance and therapeutic application. J Hematol Oncol 7: 87, 2014.

7. Mateen S, Raina K, Agarwal C, Chan D and Agarwal R: Silibinin synergizes with histone deacetylase and DNA methyltransferase inhibitors in upregulating E-cadherin expression together with inhibition of migration and invasion of human non-small cell lung cancer cells. J Pharmacol Exp Ther 345: 206-214, 2013.

8. Qu MH, Han C, Srivastava AK, Cui T, Zou N, Gao ZQ and Wang QE: miR-93 promotes TGF- $\beta$-induced epithelial-to-mesenchymal transition through downregulation of NEDD4L in lung cancer cells. Tumour Biol 37: 5645-5651, 2016.

9. Wang LK, Pan SH, Chang YL, Hung PF, Kao SH, Wang WL, Lin CW, Yang SC, Liang CH, Wu CT, et al: MDA-9/Syntenin-Slug transcriptional complex promote epithelial-mesenchymal transition and invasion/metastasis in lung adenocarcinoma. Oncotarget 7: 386-401, 2016.

10. Qu J, Li M, An J, Zhao B, Zhong W, Gu Q, Cao L, Yang H and Hu C: MicroRNA-33b inhibits lung adenocarcinoma cell growth, invasion, and epithelial-mesenchymal transition by suppressing Wnt/ß-catenin/ZEB1 signaling. Int J Oncol 47: 2141-2152, 2015.
11. Kim J, Moon SH, Kim BT, Chae CH, Lee JY and Kim SH: A novel aminothiazole KY-05009 with potential to inhibit Traf2and Nck-interacting kinase (TNIK) attenuates TGF- $\beta 1$-mediated epithelial-to-mesenchymal transition in human lung adenocarcinoma A549 cells. PLoS One 9: e110180, 2014.

12. Ebrahimi A and Sadroddiny E: MicroRNAs in lung diseases: Recent findings and their pathophysiological implications. Pulm Pharmacol Ther 34: 55-63, 2015.

13. Feng B, Zhang K, Wang R and Chen L: Non-small-cell lung cancer and miRNAs: Novel biomarkers and promising tools for treatment. Clin Sci (Lond) 128: 619-634, 2015.

14. Xia W, Chen Q, Wang J, Mao Q, Dong G, Shi R, Zheng Y, $\mathrm{Xu} \mathrm{L}$ and Jiang F: DNA methylation mediated silencing of microRNA-145 is a potential prognostic marker in patients with lung adenocarcinoma. Sci Rep 5: 16901, 2015.

15. Ju BL, Chen YB, Zhang WY, Yu CH, Zhu DQ and Jin J: miR-145 regulates chemoresistance in hepatocellular carcinoma via epithelial mesenchymal transition. Cell Mol Biol (Noisy-le-grand) 61: 12-16, 2015.

16. Tan J, Qiu K, Li M and Liang Y: Double-negative feedback loop between long non-coding RNA TUG1 and miR-145 promotes epithelial to mesenchymal transition and radioresistance in human bladder cancer cells. FEBS Lett 589: 3175-3181, 2015.

17. Zhang Y, Yang X, Wu H, Zhou W and Liu Z: MicroRNA-145 inhibits migration and invasion via inhibition of fascin 1 protein expression in non-small-cell lung cancer cells. Mol Med Rep 12: 6193-6198, 2015.

18. Ye Z, Shen N, Weng Y, Li K, Hu L, Liao H, An J, Liu L, Lao S and Cai S: Low miR-145 silenced by DNA methylation promotes NSCLC cell proliferation, migration and invasion by targeting mucin 1. Cancer Biol Ther 16: 1071-1079, 2015.

19. Livak KJ and Schmittgen TD: Analysis of relative gene expression data using real-time quantitative PCR and the 2(-Delta Delta C(T)) method. Methods 25: 402-408, 2001.

20. Sahu SK, Garding A, Tiwari N, Thakurela S, Toedling J, Gebhard S, Ortega F, Schmarowski N, Berninger B, Nitsch R, et al: JNK-dependent gene regulatory circuitry governs mesenchymal fate. EMBO J 34: 2162-2181, 2015.

21. Ding H, Ji X, Chen R, Ma T, Tang Z, Fen Y and Cai H: Antifibrotic properties of receptor for advanced glycation end products in idiopathic pulmonary fibrosis. Pulm Pharmacol Ther 35: 34-41, 2015.

22. Alizadeh AM, Shiri S and Farsinejad S: Metastasis review: From bench to bedside. Tumour Biol 35: 8483-8523, 2014.

23. Guan X: Cancer metastases: Challenges and opportunities. Acta Pharm Sin B 5: 402-418, 2015.

24. Qi D, Kaur Gill N, Santiskulvong C, Sifuentes J, Dorigo O, Rao J, Taylor-Harding B, Ruprecht Wiedemeyer W and Rowat AC: Screening cell mechanotype by parallel microfiltration. Sci Rep 5: 17595, 2015.

25. Zheng $\mathrm{H}$ and Kang Y: Multilayer control of the EMT master regulators. Oncogene 33: 1755-1763, 2014.

26. Satelli A and Li S: Vimentin in cancer and its potential as a molecular target for cancer therapy. Cell Mol Life Sci 68: 3033-3046, 2011.

27. Zhou J, Wang J, Zeng Y, Zhang X, Hu Q, Zheng J, Chen B, Xie B and Zhang WM: Implication of epithelial-mesenchymal transition in IGF1R-induced resistance to EGFR-TKIs in advanced non-small cell lung cancer. Oncotarget 29: 44332-44345, 2015.

28. Yao Y, Shi M, Liu S, Li Y, Guo K, Ci Y, Liu W and Li Y: MARVELD1 modulates cell surface morphology and suppresses epithelial-mesenchymal transition in non-small cell lung cancer. Mol Carcinog 55: 1714-1727, 2016.

29. Zhao Q, Yue J, Zhang C, Gu X, Chen $\mathrm{H}$ and Xu L: Inactivation of M2 AChR/NF- $\mathrm{BB}$ signaling axis reverses epithelial-mesenchymal transition (EMT) and suppresses migration and invasion in non-small cell lung cancer (NSCLC). Oncotarget 6: 29335-29346, 2015.

30. Ding W, Tan H, Zhao C, Li X, Li Z, Jiang C, Zhang Y and Wang L: MiR-145 suppresses cell proliferation and motility by inhibiting ROCK1 in hepatocellular carcinoma. Tumour Biol 37: 6255-6260, 2016.

31. Chen B, Huang Z, Zhang Y, Chen Y and Li Z: MicroRNA-145 suppresses osteosarcoma metastasis via targeting MMP16. Cell Physiol Biochem 37: 2183-2193, 2015.

32. Li YQ, He QM, Ren XY, Tang XR, Xu YF, Wen X, Yang XJ, Ma J and Liu N: MiR-145 inhibits metastasis by targeting fascin actin-bundling protein 1 in nasopharyngeal carcinoma. PLoS One 10: e0122228, 2015. 
33. Wang M, Wang J, Deng J, Li X, Long W and Chang Y: MiR-145 acts as a metastasis suppressor by targeting metadherin in lung cancer. Med Oncol 32: 344, 2015.

34. Wang Z, Ma B, Li H, Xiao X, Zhou W, Liu F, Zhang B, Zhu M, Yang $Q$ and Zeng Y: Protein 4.1 acts as a potential tumor suppressor linking PP1 to JNK-c-Jun pathway regulation in NSCLC. Oncotarget 7: 509-523, 2016.

35. Lo Iacono M, Monica V, Vavalà T, Gisabella M, Saviozzi S, Bracco E, Novello S, Papotti M and Scagliotti GV: ATF2 contributes to cisplatin resistance in non-small cell lung cancer and celastrol induces cisplatin resensitization through inhibition of JNK/ATF2 pathway. Int J Cancer 136: 2598-2609, 2015.

36. Song W, Ma Y, Wang J, Brantley-Sieders D and Chen J: JNK signaling mediates EPHA2-dependent tumor cell proliferation motility, and cancer stem cell-like properties in non-small cell lung cancer. Cancer Res 74: 2444-2454, 2014.
37. Luo XJ, Liu B, Dai Z, Li TB, Li NS, Zhang XJ, Yang ZC, Li YJ and Peng J: Expression of apoptosis-associated microRNAs in ethanol-induced acute gastric mucosal injury via JNK pathway. Alcohol 47: 481-493, 2013.

38. Chen R, Chen S, Liao J, Chen X and Xu X: MiR-145 facilitates proliferation and migration of endothelial progenitor cells and recanalization of arterial thrombosis in cerebral infarction mice via JNK signal pathway. Int J Clin Exp Pathol 8: 13770-13776, 2015.

39. Hu P, Huang Q, Li Z, Wu X, Ouyang Q, Chen J and Cao Y: Silencing MAP3K1 expression through RNA interference enhances paclitaxel-induced cell cycle arrest in human breast cancer cells. Mol Biol Rep 41: 19-24, 2014.

40. Wei X, Zhang E, Wang C, Gu D, Shen L, Wang M, Xu Z, Gong W, Tang C, Gao J, et al: A MAP3k1 SNP predicts survival of gastric cancer in a Chinese population. PLoS One 9: e96083, 2014. 